Ein genügendes Resultat gibt die Bestimmung des Zinks als Zinksulfid nur dann, wenn man dasselde nach dem Trocḱnen zerreibt und mit Schwefel im Rose'schen Tiegel gut mengt. Auf diese Weise wurden $68,97 \%$ Zink gefunden. Aber diese Operation erfordert peinliche Accuratesse und trotzdem können Partikel des harten Schwefelzinks beim Zerreiben wegspringen. Ferner muss berücksichtigt werden, dass der Schwefel meist etwas feuerbeständige Substanz enthält, welche bestimmt und in Abzug gebracht werden muss. Man hat also den mit dem Zinksulfid zu mengenden Schwefel vorher zu wiegen. Der von mir angewandte, rein sein sollende Schwefel enthält z. B. $0,356 \%$ einer erdigen, braunen, Eisenoxyd führenden Substanz. Da aber das Eisen darin ebenfalls sulfurirt wird, so entsteht daraus ein kleiner Febler, welcher jedoch wohl unberücksichtigt bleiben kann.

Löst man das geglühte Schwefelzink in Königswasser, so bleibt eine nicht zu vernachlässigende Menge Substanz (hauptsächlich Kieselsäure) zurück, welche stets in Abzug gebracht werden muss, da sie mehrere Zehntel Procent ausmachen kann. Ueberdies weiss man nicht, wie viel dieselbe von der feuerbeständigen Verunreinigung des Schwefels enthält, die man aber bereits abgezogen hat. Streng genommen müsste man deshalb auch noch den in Königswasser unlöslichen Bestandtheil der erdigen Verunreinigung des Schwefels ermitteln und bei der Bestimmung des Zinks in Berücksichtigung ziehen. Man sieht also, dass die Bestimmung des Zinks als Schwefelzink bei ganz genauen Analysen keinen Zeitgewinn bringt und dass in diesen Fällen die Ermittelung aus Zinkoxyd als die bequemere und sicherere vorzuziehen ist.

Hamburg, Lahoratorium des Verfassers, im August 1885.

Neue Farbenreactionen einiger Alkaloide. Von

\title{
W. Lenz.
}

Als ich im Jahre 1882 zur Identificirung von Aloëtin mit Chrysophansäure die von Liebermann angegebene Farbenreaction der Kalischmelze heranzog*), wurden auch eine Anzahl anderer organischer Körper, und besonders Alkaloide, auf ihr Verhalten gegen schmelzendes Kalihydrat geprüft. Die gemachten Wahrjehmungen veranlassten mich,

*) Diese Zeitschrift 21, 226. 
meinen damaligen Assistenten, Herrn H. S a $\mathrm{z}$ m a n n, die gefundenen Reactionen bei den 72 Alkaloiden einer von Dr. Th. S chu char d t in Görlitz erhaltenen Alkaloidsammlung durchprobiren zu lassen. Die hierbei gewonnenen positiven Resultate habe ich dann nochmals, theilweise unter Heranziehung von Präparaten verschiedener Abstammung, bearbeitet.

In erster Linie handelte es sich um Beantwortung der Frage: Welche Alkaloide geben beim Schmelzen mit Aetzkali Farbenreactionen? Schmilzt man etwas alkoholgereinigtes Aetzkali auf dem Tiegeldeckel*) mit wenig Wasser, mischt eine Spur der zu untersuchenden Substanz (am besten in alkoholischer Lösung) zu, und erhitzt nun recht vorsichtig und allmählich über einer ruhigen Flamme, schliesslich bis zur Rothgluth, so färbt sich die Masse meist gelb, allmählich in braun bis roth übergehend und verkohlt schliesslich. Diese Färbungen waren in keiner Weise auffallend ${ }^{* *}$ ) oder charakteristisch bei folgenden Körpern:

Aconitin (sowohl aus A. Napellus wie aus A. ferox und aus japanischen Knollen) Anemonin, Aspidospermin (amorph und krystallisirt) Atropin, Baptisin, Bebeerin, Berberin, Bryonin, Brucin, Caffein, Cannabin. tannicum, Cantharidin, Chelidonin, Chlorogenin, Codeïn, Colchicin, Colocynthin, Colocynthidin, Coniin. hydrobromic., Cotoïn (vernm und para), Cryptopin, Curarin. sulf., Cyclamin, Daturin, Delphinin, Digitalin, Ditaïn, Duboisin, Elaterin, Emetin, Erythrophlaeïn. hydrochlor., Gelsemin, Gentisin, Helleboreïn, Hydrocotoin, Homatropin, Hyoscyamin, Hydrastin, Hyoscin. hydrojod., Kosin, Leptandrin, Lobeliin, Lycoctonin, Meconin, Morphin, Napellin, Narceïn, Narcotin, Papaverin, Papayotin, Pelletierin. sulf., Peucedanin, Physostigmin. salicyl., Pilocarpin. hydrochloric., Piperin, Podophyllotoxin, Sabbatin, Scillitoxin, Scoparin, Solanin, Sparteïn, Strychnin, Veratrin.

Dagegen trat bei Chinin und Chinidin eine intensiv grasgrïne, bei Cinchonin und Cinchonidin eine blaugrüne, bei Cocaïn eine grụ̈nlichgelbe, bei weiterem Erhitzen in's Bläuliche, dann in schmutzig rosenroth übergehende Färbung in charakteristischer Weise ein.

*) Zur Ausführung von Farbenreactionen der Alkaloide bei gerichtlich-chemischen Untersuchungen, insbesondere solchen, bei welchen erhitzt wird, benutze ich seit Jahren stets Tiegeldeckel, deren Purzellanöse abgekniffen ist, und kann dieses in jedem frequenten Läboratorium stets disponible Substrat nur bestens empfehlen.

**) Man hüte sich vor zufälligen Verunreinigungen, welche Täuschungen veranlassen können, z. B. des Kalihydrates durch Korkstückchen ete. 
Apomorphin, Sabadillin, Thebaïn zeigten anfangs schwache Grünfärbung, die später in gelbbraun überging und mit den charakteristischen Färbungen der Chinaalkaloide nicht zu verwechseln war. Ein vor mehreren Jahren gekauftes Chinolin. tartaric. und ein aus derselben Quelle stammendes Chinolin. pur. zeigten zwar die bei den Chinaalkaloiden bemerkte Grünfärbung, bei Prüfung eines neverdings als reinstes Chinolin in den Handel gelangten Präparates habe ich dagegen diese Farbenreaction nicht wieder erhalten können.

Die bei der gerichtlich-chemischen Untersuchung von fünf verschiedenen Leichen und einer nach reichlicher Bakterienbildung giftig gewordenen Leberwurst*) sowohl aus sanrer wie aus alkalischer Lösung mit Aether ausgeschüttelten Ptomaïne zeigten beim Schmelzen mit Kali keine Farbenreaction.

Hiernach geben beim Schmelzen mit Aetzkali charakteristische Farbenreactionen die geprüften China-Alkaloide und allenfalls noch Cocaïn. **) Zur annähernden Schätzung der Empfindlichlkeit dieser Farbenreactionen wurden die Kalischmelzen folgendermaassen ausgeführt: Fin Stück reines Aetzkali wurde mit so wenig Wasser erhitzt, dass die erhaltene Masse im siedenden Wasserbade völlig flüssig war und bei gewöhnlicher Temperatur erstarrte. Von der verflüssigten Masse wurden mittelst eines Glasstabes Tropfen auf eine Anzahl Tiegeldeckel übertragen, mit bestimmten, in den einzelnen Versuchsreihen absteigenden Mengen möglichst concentrirter alkoholischer Alkaloidlösung ***) gemischt and vorsichtig erhitzt. Hierbei zeigten sich folgende Erscheinungen:

Chinin, ${ }^{1 / 2} m g$, färbt die Kalischmelze grasgrün; dabei tritt ein

*) Dieselbe batte den Tod zweier Arbeiter verursacht.

**) Die Gelbfärbung der Schmelze tritt selbst bei minimalsten Mengen Cocaïn sicher ein, auch bräunt sich dieses Gelb stets; die Erzielung der für Cocain eigentlich charakteristischen Rosafärbung, welche meist nicht über die ganze Schmelze sich ausdehnt, ist jedoch schwierig und misslingt vielfach, auch tritt diese Färbung oft so schwach auf, dass sie nur bei grosser Anfmerksamkeit wahrgenommen wird. Bisweilen erzielt man mit geringen Alkaloidmengen eine intensivere Rosafärbung, als mit grösseren; regelmässig erhielt ich die Rosafärbung noch schwach mit 1/2 mg Cocaĩn. Den Grund für diese Unsicherheit im Auftreten der bezeichneten Färbung habe ich trotz Prüfung verschiedener Präparate (Cocaïnum pur. und hydrochloricum) nicht ermitteln können.

**) Zweckmässig wird der Deckel mit Kali vor dem Zumischen dieser Lösung etwas erwärmt, damit der Alkohol rascher verdunstet. 
32 Strohmer: Ein neuer Absorptionsapparat für Kohlensäurebestimmungen.

intensiver, sehr angenehmer, aromatischer (Spiraea-) Gernch auf, der für Chinin charakteristisch zu sein scheint.*)

Chinidin, $1 \mathrm{mg}$, färbt die Kalischmelze intensiv grasgrün, die Färbung wird bei weiterem Erhitzen mehr gelb, schliesslich bräunlich; noch bei $1 / 2 m g$ tritt deutliche grasgrüne Färbung der Schmelze auf.

Cinchonin, $1 \mathrm{mg}$, färbt die Spitzen des erstarrten Kalis erst rothbraun bis violettblau, die Ränder grau; später färbt sich das Kali blaugrün (Entwickelung eines etwas stechenden Geruches). Die Reaction ist noch bei $1 / 2 m g$ deutlich.

Cinchonidi n, $1 \mathrm{mg}$, färbt die Kalischmelze bräunlichroth, sodann von den Rändern anfangend blau; $1 / 2 \mathrm{mg}$ färbt noch vorübergehend, aber deutlich blau, schliesslich grau.

Bei dem Mangel an Farbenreactionen der Chinaalkaloide dürften die beschriebenen Reactionen, besonders bei gerichtich-chemischen Untersuchungen, wo nach medikamentöser Anwendung des Chinins etc. leicht ein anderes Alkaloid vorgetäuscht werden könnte, nicht ohne Werth sein. Leider bin ich durch die Berufung in meine jetzige Stellung verhindert, die angegebenen Reactionen näher zu studiren und besonders in der Kalischmelze noch reducirende oder oxydirende Agentien wirken zu lassen. Möge das Thema denn von anderer Seite in Angriff genommen werden.

Berlin, im October 1885.

lin neuer Absorptionsapparat für Kohlensäurebestimmangen.

$$
\text { Von }
$$

\section{F. Strohmer,}

\section{Assistent der k. k. landw. chem. Versuchsstation in Wien.}

Hat man grössere Mengen von Kohlensäure zu bestimmen, so ereignet es sich nicht selten, dass die zum Auffangen des Gases verwendeten Natronkalkröhren durch die starke Erhitzung springen und man greift dann immer wiederum zum Kugelapparate zurück, obwohl

*) Das Auftreten eines Geruches wurde bei vielen der geprüften Körper bemerkt. Zum Theil war der Geruch jedoch nicht definirbar, zum Theil z. B. bei Chinidin, Cinchonin, Cinchonidin - war es unverkennbar der Gerueb des Chinolins. 